\title{
Fairness and fuzzy coalitions
}

\section{Chiara Donnini ${ }^{1} \cdot$ Marialaura Pesce $^{2}$}

Accepted: 9 May 2021 / Published online: 2 June 2021

(C) The Author(s) 2021

\begin{abstract}
In this paper, we study the problem of a fair redistribution of resources among agents in an exchange economy á la Shitovitz (Econometrica 41:467-501, 1973), with agents' measure space having both atoms and an atomless sector. We proceed by following the idea of Aubin (Mathematical methods of game economic theory. North-Holland, Amsterdam, New York, Oxford, 1979) to allow for partial participation of individuals in coalitions, that induces an enlargement of the set of ordinary coalitions to the so-called fuzzy or generalized coalitions. We propose a notion of fairness which, besides efficiency, imposes absence of envy towards fuzzy coalitions, and which fully characterizes competitive equilibria and Aubin-core allocations.
\end{abstract}

Keywords Fairness · Fuzzy coalitions · Equal-income Walrasian allocations · Mixed markets

\section{Introduction}

In an exchange economy a redistribution of resources is considered fair if it is efficient and envy-free, that is if it is not possible to make everybody better off and none prefers the bundle of anybody else rather than to consume her own bundle. It is well known that, once agents have initially equal income, competitive allocations are fair and the

We gratefully acknowledge the Editor and the Referees for their careful reading of the paper, comments and suggestions.

Marialaura Pesce

marialaura.pesce@unina.it

Chiara Donnini

chiara.donnini@uniparthenope.it

1 Dipartimento di Studi Aziendali e Quantitativi, Università degli Studi di Napoli Parthenope, Naples, Italy

2 Dipartimento di Scienze Economiche e Statistiche, Università degli Studi di Napoli Federico II and CSEF, Naples, Italy 
reverse is not true. Furthermore, core allocations might not be fair, not even if so is the initial endowment.

Zhou (1992) introduces a stronger fairness concept, called strict fairness, by imposing, besides efficiency, that no agent envies the possibility to join a coalition and to get its average bundle. He proves that, when the set of agents is replicated, the set of strictly fair allocations of the replica economy shrinks to the set of equal-income competitive allocations, whereas, in an atomless economy, both sets coincide without asymptotic limit arguments. ${ }^{1}$ This coincidence fails in the so-called mixed markets, in which a continuum of negligible traders interacts with a family of influential traders represented by atoms of the measure space of agents.

In this paper we propose a weakening of the strict fairness notion that characterizes equal-income competitive allocations in presence of non-negligible traders. Our approach consists in enlarging the class of potentially envied coalitions and following the idea of Aubin (1979) to allow every agent to choose her rate of participation in a coalition, and not only whether to participate or not. We propose a fairness notion that requires efficiency and absence of envy towards the so-called fuzzy (or generalized) coalitions. In other words, we define an efficient allocation to be fuzzy strictly fair if almost every agent $t$ does not envy a fuzzy coalition, that is if it is not possible for a certain group of traders to redistribute among themselves their resources according to their participation rates and get a bundle that $t$ strictly prefers to her own.

We show that fuzzy strict fairness $F S F$ has the twofold merit to characterize the set of equal-income competitive allocations $W_{e i}$, and simultaneously, the fuzzy core or the Aubin-core $C_{A}$ of an economy that may exhibit atoms (Theorem 3.4 and Corollary 3.5). This is contrary to the strict fairness $S F$ notion of Zhou (1992) which makes this job only under perfect competition. As a matter of fact, we prove that, in atomless economies, $S F$ and $F S F$ coincide (Corollary 4.2), and hence, the equivalence theorem of Zhou (1992) follows from ours. But, in general, FSF is a proper subset of $S F$, because an enlargement of the set of potentially envied coalitions causes the reduction of the set of allocations judgeable fair (Proposition 2.5).

Moreover, we investigate the properties of envied fuzzy coalitions and get further characterizations of competitive equilibria and of the Aubin-core. Thanks to the Lyapunov-Richter's theorem, we observe that if negligible agents are members of an envied coalition, they join it with their whole resources (Proposition 4.1). Hence, the opportunity to employ a fraction of their endowment is actually significant only for the non-negligible traders (atoms). This possibility also allows an individual to belong simultaneously to the set of envious agents and to the envied coalition. Indeed, we prove that once a trader envies a fuzzy coalition, almost every agent in the economy joins the envied coalition (Theorem 4.3). This means that envied fuzzy coalitions have full support in the sense that they are non-null almost everywhere.

Azrieli and Lehrer (2007) point out two main interpretations of fuzzy coalitions in the literature. The first is, as already observed, to let agents choose their level of participation in a coalition. The second, suggested by García-Cutrín and Hervés-Beloso (1993) and later by Hüsseinov (1994), consists in associating to every finite economy an atomless one and interpreting fuzzy coalitions in the finite economy as ordinary

\footnotetext{
${ }^{1}$ See also Thomson (1988) and Thomson (2011).
} 
coalitions in the associated atomless economy. We extend this interpretation to mixed markets in terms of envied coalitions rather than blocking coalitions. Precisely, given a mixed economy $\mathcal{E}$, we consider the atomless economy $\mathcal{E}^{*}$ as determined by Greenberg and Shitovitz (1986), and we show that there is a one-to-one correspondence between the sets $F S F$ of $\mathcal{E}$ and $S F$ of $\mathcal{E}^{*}$. Indeed, we prove that any fuzzy strictly fair allocation in the mixed economy $\mathcal{E}$ corresponds to a unique strictly fair allocation in $\mathcal{E}^{*}$ and vice-versa. This allows us to view fuzzy coalitions and fuzzy strictly fair allocations respectively as standard coalitions and strictly fair allocations of an associated, suitably defined, atomless economy.

Summing up, our analysis contributes to two pieces of literature, the one that studies the problem of a fair redistribution of divisible resources and the other that concerns the fuzzy cooperation approach and possible characterizations of competitive equilibria in non-perfectly competitive economies. The literature of fair division is huge and rich of various equity notions proposed in decades of research. This paper focuses on the notion of strict fairness due to Zhou (1992) which has been recently studied by Cato (2010), who proposed a local version of $S F$ by imposing absence of envy only among neighbors (see also Donnini and Pesce 2021), by Basile et al. (2018) who compared $S F$ with a coalitional fairness notion in economies with infinitely many goods, by Donnini and Pesce (2020) who got the equivalence $W_{e i}=S F$ in mixed markets under stronger assumptions than those used to prove the Core-Walras equivalence theorem (Shitovitz 1973). In this paper, we keep the model of mixed markets but, weakening the notion of strict fairness, we avoid additional conditions on the space of agents as well as restrictions on the set of potentially envied agents. We indeed follow Aubin's approach which permits extending several results to non-perfectly competitive economies. This policy is also widespread in the literature. See, among others, Florenzano (1990), Noguchi (2000), Liu and Liu (2014), Pesce (2014) and Bhowmik and Graziano (2015) for the equivalence Walras-Aubin-Core in different frameworks; Hervés-Estévez and Moreno-García (2015), Hervés-Beloso et al. (2018) and Graziano et al. (2020) for the link between $W_{e i}$ and the bargaining set, and Yang et al. (2011) between the bargaining set and the Aubin-core.

The paper is organized as follows. In Sect. 2 we describe the model, we introduce the notion of fuzzy strict fairness, and we provide some basic relationships among the main definitions. Section 3 contains the equivalence theorem, whereas Sect. 4 some properties of fuzzy envied coalitions and consequent characterizations of the set of fuzzy strictly fair allocations. Section 5 refers to the construction of the atomless economy $\mathcal{E}^{*}$ associated to the mixed market $\mathcal{E}$ and provides an interpretation of fuzzy coalitions and of the set of fuzzy strictly fair allocations. Section 6 concludes with some remarks.

\section{The economic model and the main definitions}

We consider an exchange economy $\mathcal{E}$ with a finite number $\ell$ of different commodities. The commodity space is $\mathbb{R}_{++}^{\ell}$, the interior of the positive orthant of the Euclidean 
space $\mathbb{R}^{\ell}$, which contains the total initial endowment of the economy $\mathcal{E}$ denoted by $e .^{2}$ The agents are represented as the points of a $\sigma$-additive, complete probability space $(T, \Sigma, \mu)$, and as usual, a coalition is any element of $\Sigma$ with positive measure. Each agent $t \in T$ has a utility function $u_{t}: \mathbb{R}_{+}^{\ell} \rightarrow \mathbb{R}$, representing her preferences, which is assumed to be continuous, strictly monotone and quasi-concave. ${ }^{3}$ Furthermore, the usual measurability condition is required, that is the map $(t, x) \rightarrow u_{t}(x)$ is $\Sigma \otimes$ $\mathcal{B}\left(\mathbb{R}_{+}^{\ell}\right)$-measurable, where $\mathcal{B}\left(\mathbb{R}_{+}^{\ell}\right)$ is the $\sigma$-field of Borel subsets of $\mathbb{R}_{+}^{\ell}$. For the equivalence theorem and its corollary only, we also need that $u_{t}(\cdot)$ is differentiable for all $t \in T$ (see Zhou 1992; Thomson and Zhou 1993). Since we do not require $\mu$ to be non-atomic, it is allowed the presence in $\Sigma$ of $\mu$-atoms, that are sets $A \in \Sigma$ with nonzero measure such that $\mu(A \backslash B)=0$ or $\mu(B)=0$ for every other $B \subseteq A$. According to the atomless-atomic decomposition of measures, $T$ is partitioned into an atomless set $T_{0}$ (atomless component) representative of an ocean of negligible traders, and a set $T_{1}=T \backslash T_{0}$, which is the union of an at most countable family $\left\{A_{1}, A_{2}, \ldots, A_{k}, \ldots\right\}$ of disjoint atoms representative of non-negligible traders. Each atom can be interpreted as a single individual concentrating in her hands a large amount of the total initial endowment (oligopolistic agent) or as a group of individuals deciding to act only together (cartels, syndicates). Abusing notation, we use the same symbol $T_{1}$ to denote the collection $\left\{A_{1}, A_{2}, \ldots, A_{k}, \ldots\right\}$ as well, and we write $A \in T_{1}$ instead of $A \subseteq T_{1}$. Two agents $t, s$ are said to be of the same type or identical if they have the same utility function $u_{t}=u_{s} .{ }^{4}$ Two identical atoms are said to be of the same kind if they have the same measure too. A measurable mapping is almost everywhere constant on an atom, then the measurability condition imposed on the utility function ensures that for every $A \in T_{1}$ and $t, s \in A$ we have $u_{t}=u_{s}$, that is $t$ and $s$ are of the same type.

This model includes as particular case different situations. The framework of a discrete economy is covered by a finite set $T$ with a counting measure $\mu$, whereas the case of perfect competition by the Lebesgue measure space $(T, \Sigma, \mu)$, with $T=[0,1]$. Finally, when both sets $T_{0}$ and $T_{1}$ have positive $\mu$-measure, $\mathcal{E}$ is called mixed market or mixed economy and it allows interaction between an ocean of negligible agents and at most countably many atoms that are the influential agents or oligopolies.

An allocation is a $\mu$-integrable ${ }^{5}$ function $x: T \rightarrow \mathbb{R}_{++}^{\ell}$ assigning to each agent $t \in T$ her bundle $x(t) \in \mathbb{R}_{++}^{\ell}$. An allocation $x$ is said to be feasible if its aggregate equals the total initial endowment, i.e. $\int_{T} x(t) d \mu(t)=e$. A feasible allocation $x$ is efficient or Pareto optimal if it does not exist an alternative feasible allocation $y$ such that $u_{t}(y(t))>u_{t}(x(t))$ almost everywhere in $T$. A feasible allocation $x$ is competitive or

\footnotetext{
2 The commodity space can be enlarged to $\mathbb{R}_{+}^{\ell}$ provided that additional conditions on the primitives of the economy $\mathcal{E}$ are imposed. We refer to Remark 2.2 for details.

${ }^{3}$ Strict monotonicity means that $y>x \Rightarrow u(y)>u(x)$, and when $u$ is also differentiable that $\nabla u(x)>0$ for all $x \in \mathbb{R}_{++}^{\ell}$. The function $u$ is quasi-concave if $u(\alpha x+(1-\alpha) y) \geqslant \min \{u(x), u(y)\}$ for any $x, y \in$ $\mathbb{R}_{++}^{\ell}$ and any $\alpha \in[0,1]$.

${ }^{4}$ In Shitovitz (1973), identical agents must also have the same initial endowment. In our framework, as in most of the papers dealing with fairness, it is irrelevant how the total initial endowment $e$ is distributed among traders. However, being $\mu(T)=1$, the vector $e$ may also denote the initial endowment of any single individual $t \in T$. In other words, we may consider the constant function $e: T \rightarrow \mathbb{R}_{++}^{\ell}$ as $e(t)=e$ for all $t \in T$.

5 The integral of a vector function is the vector of integrals of the components.
} 
Walrasian if there exists a price vector $p \gg 0$ such that for almost all agent $t \in T$, $p \cdot x(t) \leqslant p \cdot e$ and $p \cdot y>p \cdot e$ whenever $u_{t}(y)>u_{t}(x(t))$. We denote by $W_{e i}$ the set of equal-income competitive allocations of $\mathcal{E}$. Following Aubin (1979), we allow agents to join a coalition using only a fraction of their endowment. The idea is to assign to each individual $t$ a real number $\gamma(t) \in[0,1]$ representing her personal portion of resources she wants to invest into a coalition. Formally, a fuzzy coalition is any couple $(\gamma, S)$ where $\gamma: T \rightarrow[0,1]$ is a non-null simple and measurable function and $S$ is its support, i.e. the set $\{t \in T: \gamma(t)>0\}$ with $\mu(S)>0 .{ }^{6}$ We denote by $\mathcal{F}$ the collection of all fuzzy coalitions, and we observe that any element $S$ of $\Sigma$, called throughout the paper standard or crisp coalition, can be paired with its correspondent characteristic function ${ }^{7} \chi_{S}$ and it can be considered as a fuzzy coalition in $\mathcal{F}$. Therefore, we enlarge the class of standard coalitions since $\Sigma$ can be viewed as a subset of $\mathcal{F}$, i.e. $\Sigma \subseteq \mathcal{F}$. A fuzzy coalition $(\gamma, S)$ is said to have full support if $\gamma(t)>0$ for almost all $t \in T$ and hence $\mu(S)=\mu(T)=1$. An allocation is blocked by a fuzzy coalition $(\gamma, S) \in \mathcal{F}$ if there exists an alternative allocation $y$ such that $u_{t}(y(t))>u_{t}(x(t))$ for almost all $t \in S$ and $\int_{S} \gamma(t) y(t) d \mu(t)=\int_{S} \gamma(t) e d \mu(t)$. A feasible allocation $x$ is in the Aubin core of the economy $\mathcal{E}$, denoted by $C_{A}$, if it is not blocked by any fuzzy coalition. If $x$ is not blocked by any crisp coalition, then it is in the core of the economy $\mathcal{E}$, which is denoted by $C$. Thus, in the light of the inclusion $\Sigma \subseteq \mathcal{F}$, it is clear that $C_{A} \subseteq C$. Furthermore, it is known that in a mixed economy, under standard assumptions, Walrasian equilibria are the only allocations in the Aubin core (see for example Aubin 1979; Florenzano 1990; Noguchi 2000), whereas there are core allocations that are not competitive, unless stronger assumptions on the space of agents are imposed (see Shitovitz 1973; Gabszewicz and Mertens 1971; Greenberg and Shitovitz 1986 among the others). Then, $W_{e i}=C_{A} \subseteq C$.

The first notion of fairness is due to Foley (1967) for which an allocation $x$ is said to be fair if it is efficient and envy-free, meaning that every agent $t$ prefers to consume her own bundle rather than receive the bundle of anybody else, i.e. $u_{t}(x(t)) \geqslant u_{t}(x(s))$ for all $s \in T$. An equal-income competitive allocation is fair, but the converse is not true. Our goal is to propose a notion of fairness that fully characterizes equalincome competitive allocations, and consequently the Aubin core, in the very general framework of mixed markets.

Definition 2.1 Given an allocation $x$, an agent $t$ envies a fuzzy coalition $(\gamma, S)$ at $x$ if there exists an alternative allocation $y$ for which

$$
\begin{aligned}
& u_{t}(y(s))>u_{t}(x(t)) \text { for almost every } s \in S, \\
& \int_{S} \gamma(s) y(s) d \mu(s)=\int_{S} \gamma(s) x(s) d \mu(s) .
\end{aligned}
$$

Condition (1) means that an agent $t$ prefers the alternative bundle $y(s)$ received by any member $s$ of $S$ rather than to consume her own bundle $x(t)$; by (2) agents join the fuzzy coalition $(\gamma, S)$ by employing only a share of their resources and redistribute

\footnotetext{
6 The same notation is used in Graziano et al. (2020).

7 For any $S \in \Sigma$, the characteristic function of $S$ is the function $\chi_{S}: T \rightarrow\{0,1\}$ that assigns 1 to each $t \in S$ and 0 to every other $t$ outside $S$. Then, $\left(\chi_{S}, S\right) \in \mathcal{F}$.
} 
among themselves their bundles $x$ according to these shares. An allocation $x$ is fuzzy strictly envy-free (or fuzzy strictly equitable) if almost every agent $t$ is not envious, that is $t$ does not envy any fuzzy coalition. An allocation is said to be fuzzy strictly fair if it is both fuzzy strictly envy-free and efficient. The set of fuzzy strictly fair allocations is denoted by $F S F$.

Remark 2.2 For the proof of the equivalence theorem it is crucial that any allocation $x$ in $F S F$ has positive components, which is true because $x \in \mathbb{R}_{++}^{\ell}$. However, the commodity space can be enlarged to $\mathbb{R}_{+}^{\ell}$ and any $x \in F S F$ is still strictly positive if $e \gg 0$ and if we impose the boundary condition, that is for each $x \in \partial \mathbb{R}_{+}^{\ell}$ and $y \in \mathbb{R}_{++}^{\ell}, u_{t}(x)<u_{t}(y)$. Indeed, if $x \in F S F$ and $x(t) \in \partial \mathbb{R}_{+}^{\ell}$ for all $t$ in a certain coalition $S$ of $\Sigma$, being $e \gg 0$, we have that $u_{t}(e)>u_{t}(x(t))$ for all $t \in S$. Thus, since $x$ is feasible, any member of $S$ envies the fuzzy coalition $(1, T)$, which is impossible because $x \in F S F$.

\subsection{Basic relationships}

In this section we compare Definition 2.1 with the notion of strict fairness due to Zhou (1992) that we recall below.

Definition 2.3 [Strict fairness of Zhou (1992)] Given an allocation $x$, an agent $t$ envies a crisp coalition $S$, with $t \notin S$, if $u_{t}(\bar{x}(S))>u_{t}(x(t))$, where $\bar{x}(S)=$ $\frac{1}{\mu(S)} \int_{S} x(s) d \mu(s)$. An allocation $x$ is strictly envy-free (or strictly equitable) if almost every agent does not envy any (standard) coalition. An allocation is said to be strictly fair if it is both strictly envy-free and efficient. The set of strictly fair allocations is denoted by $S F$.

Definition 2.3 strengthens the notion of average A-fairness of Thomson (1982) according to which each individual weakly prefers her own bundle to the average of what all the others receive, i.e. $S=T \backslash\{t\}$ in Definition 2.3 (see also Thomson 1988). In a two-agent economy the fairness concepts of Foley (1967), Thomson (1982) and Zhou (1992) are equivalent, but in general strict fairness (Definition 2.3) is the strongest one, whereas average fairness of Thomson (1982) and the fairness notion of Foley (1967) are not comparable (see Proposition 1 in Thomson 1982).

Since $\Sigma \subseteq \mathcal{F}$ there are more coalitions that each individual can envy and then, fewer allocations pass the test of fairness. Consequently, the set of fuzzy strictly fair allocations is included into the set of strictly fair allocations, whereas the converse is not true as shown in Proposition 2.5 for which the next lemma is needed.

Lemma 2.4 Given an allocation $y$, a vector $k \in \mathbb{R}_{+}^{\ell}$ and a coalition $S$ such that $u(y(s))>u(k)$ for almost all $s \in S$, then $u\left(\frac{1}{\mu(S)} \int_{S} y(s) d \mu(s)\right)>u(k)$. The implication holds also with " $\geqslant$ " instead of " >".

Proof See Lemma in García-Cutrín and Hervés-Beloso (1993) and Lemma 7.1 in Basile et al. (2018). ${ }^{8}$

\footnotetext{
${ }^{8}$ Remember that throughout the paper $u$ is assumed continuous, strictly monotone and quasi-concave.
} 
Proposition 2.5 The set of fuzzy strictly fair allocations is included into the set of strictly fair allocations, whereas the converse is not true neither in a mixed economy with $n$ atoms of the same kind, i.e., FSF $\varsubsetneqq S F$.

Proof Let $x$ be a fuzzy strictly fair allocation and assume to the contrary that $x$ is not strictly envy-free. Thanks to Lemma 2.4, an agent $t$ envies a crisp coalition $S$ at $x$ if it is possible to redistribute among members of $S$ the aggregate bundle $\int_{S} x(t) d \mu(t)$ in such a way that $t$ prefers the bundle of almost every member of $S$ to her own. This means that for any envious agent $t$ there exist an allocation $y$ and a crisp coalition $S \in \Sigma$ such that

$$
\begin{gathered}
u_{t}(y(s))>u_{t}(x(t)) \text { for almost all } s \in S, \text { and } \\
\int_{S} y(s) d \mu(s)=\int_{S} x(s) d \mu(s) .
\end{gathered}
$$

Then, $t$ envies the fuzzy coalition $(1, S)$, which is impossibile being $x \in F S F$. Hence, $F S F \subseteq S F$. In order to prove that the inclusion is strict, consider a mixed market whose consumption set is $\mathbb{R}_{++}^{2}$ and the total initial endowment is $e=(1,1) \gg 0$. Let $T=T_{0} \cup T_{1}$ where $T_{0}=\left[0, \frac{1}{2}\right]$ and $T_{1}=\left\{A_{1}, \ldots, A_{n}\right\}$ with $\mu\left(A_{i}\right)=\frac{1}{2 n}$ for every $i=1, \ldots, n$. Furthermore, let agents' utility function be given by

$$
u_{t}(x, y)= \begin{cases}x y & \text { if } t \in T_{0} \\ x^{2} y & \text { if } t \in T_{1}\end{cases}
$$

and consider the feasible allocation $(x, y)$ defined by $(x(t), y(t))=(a, b) \chi_{T_{0}}+(2-$ $a, 2-b) \chi_{T_{1}}$, where $b=\frac{(n-1) a+2}{n(2 a-1)+1}$ and $a=\frac{3 n-2+\sqrt{9 n^{2}+16 n+8}}{7 n+1}$.

It can be shown that this allocation is strictly fair (see Proposition 3.3 in Donnini and Pesce 2020 for details ${ }^{9}$ ) but, as proved below, it is not fuzzy strictly fair. To this end, consider the allocation $(c, d)$ assigning to each agent $t$ in $T$ the following bundle

$$
(c(t), d(t))=\left(2 \frac{n+1}{n+2}(a-1)+2-a, 2 \frac{n+1}{n+2}(b-1)+2-b\right),
$$

and the generalized coalition $(\gamma, T)$ defined as $\gamma(t)=1 \chi_{T_{0}}+\frac{1}{n+1} \chi_{T_{1}}$. With algebraic computation, we can write $d=2 \frac{(n+1)^{2}}{n+2} \frac{1-a}{n(2 a-1)+1}+\frac{(3 n+1) a-2 n}{n(2 a-1)+1}$ and prove that any agent $t$ in $T_{0}$ envies the fuzzy coalition $(\gamma, T)$ at $(x, y)$. Indeed, for every $t$ in $T_{0}$ and for every $s$ in $T$,

$$
\begin{aligned}
& u_{t}(x(t), y(t))=a b<c d=u_{t}(c(s), d(s)) \\
& \Longleftrightarrow a((n-1) a+2)<\left(2 \frac{n+1}{n+2}(a-1)+2-a\right)\left(2 \frac{(n+1)^{2}}{n+2}(1-a)+(3 n+1) a-2 n\right) \\
& \Longleftrightarrow(1-a)^{2}\left[(n+1)^{3}-(n+1)(n+2)(2 n+1)+n(n+2)^{2}\right]<0 \Longleftrightarrow-1<0 .
\end{aligned}
$$

\footnotetext{
9 Donnini and Pesce (2020) describe the same economy for a different purpose.
} 
Moreover,

$$
\begin{aligned}
\int_{T} \gamma(s)((c, d)-(x, y)) d \mu(s) & =\int_{T_{0}}(c-a, d-b) d \mu(s)+\int_{T_{1}} \frac{1}{n+1}(c-2+a, d-2+b) d \mu(s) \\
& =\left(\frac{-1}{n+2}(a-1), \frac{-1}{n+2}(b-1)\right)+\left(\frac{1}{n+2}(a-1), \frac{1}{n+2}(b-1)\right) \\
& =(0,0) .
\end{aligned}
$$

Thus, $(x, y) \in S F \backslash F S F$.

\section{The equivalence theorem}

Our main goal is to prove the equivalence $W_{e i}=F S F$. We first show that equalincome Walrasian allocations, and hence Aubin core allocations, are fuzzy strictly fair.

Proposition 3.1 Any equal-income Walrasian allocation $x$ is fuzzy strictly fair, i.e. $W_{e i} \subseteq F S F$.

Proof Let $x$ be an equal-income Walrasian allocation supported by the equilibrium price $p$ and assume to the contrary that $x$ is not fuzzy strictly fair. Since, by the first welfare theorem, $x$ is efficient it means that the set of agents envying a fuzzy coalition at $x$ has positive measure. Then, for each envious agent $t$ there exist a fuzzy coalition $(\gamma, S)$ and an allocation $y$ satisfying the conditions of Definition 2.1. From (1) of Definition 2.1, we have that for almost every $s$ in $S, p \cdot y(s)>p \cdot e \geqslant p \cdot x(s)$, and then $p \cdot \int_{S} \gamma(s) y(s) d \mu(s)>p \cdot \int_{S} \gamma(s) x(s) d \mu(s)$, which contradicts condition (2) of Definition 2.1.

Corollary 3.2 Any Aubin-core allocation $x$ is fuzzy strictly fair, i.e. $C_{A} \subseteq F S F$.

Proof It follows from Proposition 3.1 and the equivalence $W_{e i}=C_{A}$ proved by Florenzano (1990), Noguchi (2000) and Liu and Liu (2014) among others.

Corollary 3.2 can be further extended to the notion of bargaining set due to HervésEstévez and Moreno-García (2018a) (see also Hervés-Estévez and Moreno-García 2018b; Hervés-Beloso et al. 2018), since it coincides with the sets $W_{e i}$ and $C_{A}$ as shown by Theorem 1 in Graziano et al. (2020). Our framework ensures the existence of Walrasian equilibrium and Aubin-core allocation in mixed markets, from which, together with Proposition 3.1 and Corollary 3.2, we deduce that $F S F \neq \emptyset$. We refer to Theorem 2 of Hildenbrand (1974), page 151, for the existence of Walrasian equilibrium, whereas see, for instance, Florenzano (1990) and Allouch and Predtetchinski (2008) for $C_{A} \neq \emptyset$.

In order to prove the reverse inclusion $F S F \subseteq W_{e i}$ we need the following lemma, which can be viewed as an extension to mixed markets of Lemma 3.3 of Zhou (1992) (see also Thomson and Zhou 1993; Basile et al. 2018 for infinite-dimensional separable commodity space).

Lemma 3.3 Given an allocation $x$, 
(i) the set $X=\left\{y \in \mathbb{R}_{+}^{\ell}: y=\int_{S} \gamma(t) x(t) d \mu(t)\right.$ for some $\left.(\gamma, S) \in \mathcal{F}\right\}$ is convex, and

(ii) $x(t) \in \operatorname{cl}(X)$ for almost all $t \in T$, where $c l(X)$ denotes the closure of $X$.

Proof (i) We know that $T=T_{0} \cup T_{1}$, where $T_{0}$ is the atomless part and $T_{1}$ is the union of at most countably many disjoint atoms. Define the sets

$$
\begin{aligned}
& X_{0}:=\left\{\int_{S \cap T_{0}} \gamma(t) x(t) d \mu(t):(S, \gamma) \in \mathcal{F}\right\} \text { and } \\
& X_{1}:=\left\{\int_{S \cap T_{1}} \gamma(t) x(t) d \mu(t):(S, \gamma) \in \mathcal{F}\right\},
\end{aligned}
$$

and notice that $X=X_{0}+X_{1}$, where $X_{0}$ is convex thanks to Lyapunov-Richter's theorem. It is then enough to show that $X_{1}$ is convex too. Let $\alpha y_{1}+(1-\alpha) y_{2}$ be a convex combination of two elements $y_{1}$ and $y_{2}$ of $X_{1}$. Then, there exist two fuzzy coalitions $\left(S_{1}, \gamma_{1}\right),\left(S_{2}, \gamma_{2}\right) \in \mathcal{F}$ such that

$$
y_{1}=\int_{S_{1} \cap T_{1}} \gamma_{1}(t) x(t) d \mu(t) \text { and } y_{2}=\int_{S_{2} \cap T_{1}} \gamma_{2}(t) x(t) d \mu(t) .
$$

Define now the fuzzy coalition $\left(\gamma, S_{1} \cup S_{2}\right) \in \mathcal{F}$ as $\gamma=\left(\alpha \gamma_{1}+(1-\alpha) \gamma_{2}\right) \chi_{S_{1} \cup S_{2}}$; and notice that

$$
\alpha y_{1}+(1-\alpha) y_{2}=\int_{\left(S_{1} \cup S_{2}\right) \cap T_{1}} \gamma(t) y(t) d \mu(t) \in X_{1} .
$$

Then, the set $X_{1}$ is convex and so is $X$.

(ii) If $y \notin \operatorname{cl}(X)$, there exists a ball $O_{y}$ around $y$ such that $O_{y} \cap X=\emptyset$. Therefore, the set of agents $t$ for which $x(t) \in O_{y}$ has null measure, otherwise the integral over such a set of $x$ is in $O_{y}$ and by definition it is also in $X$, contradicting the fact that $O_{y} \cap X=\varnothing$. Therefore,

$$
\mu\left(\left\{t \in T: x(t) \in O_{y}\right\}\right)=0 .
$$

Since $\mathbb{R}_{+}^{\ell} \backslash c l(X)$ is separable, there are countably many $O_{y}$ satisfying (1) whose union covers $\mathbb{R}_{+}^{\ell} \backslash \operatorname{cl}(X)$. This implies that $\mu\left(\left\{t \in T: x(t) \in \mathbb{R}_{+}^{\ell} \backslash \operatorname{cl}(X)\right\}\right)=0$ and hence $x(t) \in c l(X)$ for almost all $t \in T$.

Lemma 3.3 consists into two statements: the former does not follow from the Lyapunov-Richter's theorem because the space of agents $(T, \Sigma, \mu)$ is not atomless. Nevertheless, the set $X$ is convex thanks to the fuzzy coalitions $(\gamma, S) \in \mathcal{F}$ that have this convexification effect. The second statement follows from the separability of the commodity space and it will be crucial in the proof of the equivalence Theorem 3.4.

Theorem 3.4 If $u_{t}$ is differentiable for all $t \in T$, then $W_{e i}=F S F$. 
Proof The inclusion $W_{e i} \subseteq F S F$ is proved in Proposition 3.1. For the converse, let $x$ be a fuzzy strictly fair allocation, i.e. $x \in F S F$. In particular, $x$ is efficient then, by the second welfare theorem, there exists a price vector $p \gg 0$ supporting $x$, that is $u_{t}(y)>u_{t}(x(t)) \Rightarrow p \cdot y>p \cdot x(t)$. This means that for almost all $t \in T, x(t) \in \operatorname{argmax}_{y \in\left\{y \in \mathbb{R}_{++}^{\ell}: p \cdot y \leq p \cdot x(t)\right\}} u_{t}(y)$. To conclude the proof we need to show that $p \cdot x(t)=p \cdot e$ for almost all $t \in T$. Denote by $C_{1}$ the set of agents $t$ for which $x(t) \notin \operatorname{argmax}_{y \in\left\{y \in \mathbb{R}_{++}^{\ell}: p \cdot y \leq p \cdot x(t)\right\}} u_{t}(y)$ and by $C_{2}$ the set of agents envying a fuzzy coalition at $x$. Since $x \in F S F$, both sets have null measure. Then, we show that $p \cdot x(t)=p \cdot e$ for all $t \in C$, where $C=T \backslash\left(C_{1} \cup C_{2}\right)$. Fom the feasibility of $x$ it follows that $e \in X$, where $X$ is defined as the set

$$
X=\left\{y \in \mathbb{R}_{++}^{\ell}: y=\int_{S} \gamma(t) x(t) d \mu(t) \text { for some }(\gamma, S) \in \mathcal{F}\right\}
$$

which is convex because of Lemma $3.3(i)$.

Assume now to the contrary that the set $B=\{t \in C: p \cdot x(t)<p \cdot e\}$ has positive measure. By applying Lemma 3.3 ( $i$ i ) to $B$ we know that for almost all $t \in B, x(t) \in \operatorname{cl}(Y)$, where $^{10}$

$$
Y=\left\{y \in \mathbb{R}_{++}^{\ell}: y=\int_{S} \gamma(t) x(t) d \mu(t) \text { for some }(S, \gamma) \in \mathcal{F}_{\mid \mathcal{B}}\right\}
$$

and clearly $Y \subseteq X$.

Forasmuch as $B \subseteq C=T \backslash\left(C_{1} \cup C_{2}\right), x(t) \in \operatorname{argmax}_{y \in\left\{y \in \mathbb{R}_{++}^{\ell}: p \cdot y \leq p \cdot x(t)\right\}} u_{t}(y)$ and $p \cdot x(t)<p \cdot e$ for any $t \in B$. Since $u_{t}$ is differentiable and strictly monotone, by the first order condition, $\nabla u_{t}(x(t))$ is a positive multiple of $p$, i.e. $\nabla u_{t}(x(t))=\lambda p$ with $\lambda>0$, and hence $\nabla u_{t}(x(t)) \cdot(e-x(t))=\lambda p \cdot(e-x(t))>0$. Consider now the differentiable one-variable function $F(\alpha)=u_{t}((1-\alpha) x(t)+\alpha e)-u_{t}(x(t))$ where $\alpha \in \mathbb{R}$. By Taylor expansion of $F$ around $\alpha=0$, there exists $R(\alpha)$ such that $\lim _{\alpha \rightarrow 0} \frac{R(\alpha)}{\alpha}=0$ and

$$
\begin{gathered}
F(\alpha)=F(0)+\alpha F^{\prime}(0)+R(\alpha), \text { that is } \\
u_{t}((1-\alpha) x(t)+\alpha e)-u_{t}(x(t))=\alpha \nabla u_{t}(x(t)) \cdot(e-x(t))+R(\alpha) .
\end{gathered}
$$

Furthermore, since $\nabla u_{t}(x(t)) \cdot(e-x(t))>0$ and $\frac{R(\alpha)}{\alpha} \rightarrow 0$, there is $\bar{\alpha} \in(0,1)$ such that $R(\bar{\alpha})>-\bar{\alpha} \nabla u_{t}(x(t)) \cdot(e-x(t))$, and then

$$
u_{t}((1-\bar{\alpha}) x(t)+\bar{\alpha} e)>u_{t}(x(t))
$$

Now since $x(t) \in \operatorname{cl}(Y)$, it follows that there exists a sequence $x_{n} \in X$ converging to $x(t)$ and $(1-\bar{\alpha}) x_{n}+\bar{\alpha} e \in X$, because $e \in X$ and $X$ is convex. Furthermore, by continuity of $u_{t}$ and (2), it follows that

\footnotetext{
$10 \mathcal{F}_{\mid \mathcal{B}}$ denotes the set of fuzzy coalitions $(\gamma, S)$ with $\gamma: B \rightarrow[0,1]$ and then $S \subseteq B$.
} 


$$
u_{t}\left((1-\bar{\alpha}) x_{n}+\bar{\alpha} e\right)>u_{t}(x(t))
$$

for $n$ big enough. Fix some $\bar{n}$ for which (3) holds and, being $(1-\bar{\alpha}) x_{\bar{n}}+\bar{\alpha} e \in X$, there exists $(\gamma, S) \in \mathcal{F}$ such that

$$
(1-\bar{\alpha}) x_{\bar{n}}+\bar{\alpha} e=\int_{S} \gamma(t) x(t) d \mu(t) .
$$

Define for each $s \in S$ the bundle $y(s)=\frac{(1-\bar{\alpha}) x_{\bar{n}}+\bar{\alpha} e}{\gamma(s) \mu(S)}$ which is well-defined because $\gamma$ is simple, $\gamma(s)>0$ for any $s \in S$ and $\mu(S)>0$. Furthermore, since both $\gamma(s) \leqslant 1$ and $\mu(S) \leqslant 1$, by monotonicity and (3) it follows that $u_{t}(y(s)) \geqslant u_{t}((1-$ $\left.\bar{\alpha}) x_{\bar{n}}+\bar{\alpha} e\right)>u_{t}(x(t))$ for all $s \in S$. Finally, from (4), we have that $\int_{S} \gamma(t) y(t) d \mu(t)=$ $(1-\bar{\alpha}) x_{\bar{n}}+\bar{\alpha} e=\int_{S} \gamma(t) x(t) d \mu(t)$. This means that almost every member $t$ of $B$ envies a fuzzy coalition, which is impossible because $B \subseteq C=T \backslash\left(C_{1} \cup C_{2}\right)$ and $t$ cannot be envious being not in $C_{2}$. Hence, $\mu(B)=0$ and since $x$ is feasible, we have that $p \cdot x(t)=p \cdot e$ for almost all $t \in T$.

In atomless economies, Theorem 3.4 follows from Proposition 3.4 of Zhou (1992), whereas in the presence of non-negligible agents, in the light of Proposition 2.5, we have that $W_{e i}=F S F \varsubsetneqq S F .{ }^{11}$ As observed by Zhou (1992) we cannot dispense with the differentiability of the agents' utility functions but, at the same time, no assumption on the measure space of agents is required.

Corollary 3.5 If $u_{t}$ is differentiable for all $t \in T$, then

(1) $W_{e i}=C_{A}=F S F \varsubsetneqq S F$.

(2) If, in addition, the economy $\mathcal{E}$ is atomless, then $W_{e i}=C_{A}=C=F S F=S F$.

Actually from Theorem 1 of Graziano et al. (2020) it follows that Corollary 3.5(1) can be extended to the bargaining set as defined by Hervés-Estévez and Moreno-García (2018a) (see also Hervés-Estévez and Moreno-García 2018b; Hervés-Beloso et al. 2018) and Corollary 3.5(2) also to the bargaining set defined by Mas-Colell (1989) (see Liu and Zhang 2016 for the equivalence in coalition production economies).

\section{Properties of envied fuzzy coalitions}

In this section, we analyse some properties of envied fuzzy coalitions and we provide further characterizations of equal-income Walrasian equilibria and Aubin-core allocations.

Thanks to Lyapunov-Richter's theorem we show that if negligible agents are members of a fuzzy envied coalition, they employ the totality of their endowment. This means that the possibility of partial participation is relevant only for non-negligible agents. Similar considerations have been done by Basile et al. (2018) for coalitional fair allocations and by Graziano et al. (2020) for the bargaining set.

\footnotetext{
${ }_{11}$ Recently Donnini and Pesce (2020) prove the equivalence $W_{e i}=S F$ in mixed economies under stronger assumptions, not needed for the fuzzy strict fairness notion.
} 
Proposition 4.1 If negligible agents belong to a fuzzy envied coalition, they join it with their whole endowment.

Proof Let $(\gamma, S)$ be a fuzzy envied coalition by a certain agent $t$ at $x$ via an allocation $y$. This means that $u_{t}(y(s))>u_{t}(x(t))$ for almost all $s \in S$ and $\int_{S} \gamma(s) y(s) d \mu(s)=\int_{S} \gamma(s) x(s) d \mu(s)$. Since $\gamma$ is simple, let $\gamma_{1}, \ldots, \gamma_{k}$ be the values it takes and define, for each $i=1, \ldots, k$, the set $S_{i}=\left\{s \in S: \gamma(s)=\gamma_{i}\right\}$. By Lyapunov-Richter's theorem, for each $S_{i} \cap T_{0}$ there exists $B_{i} \subseteq S_{i} \cap T_{0}$ such that $\int_{B_{i}}(x(s)-y(s)) d \mu(s)=\gamma_{i} \int_{S_{i} \cap T_{0}}(x(s)-y(s)) d \mu(s)$. Let $(\tilde{\gamma}, B)$ be a fuzzy coalition where $B=\bigcup_{i=1}^{k} B_{i} \cup\left(S \cap T_{1}\right)$ and $\tilde{\gamma}=\left.1 \chi\right|_{B \cap T_{0}}+\gamma \chi_{S \cap T_{1}}$. Notice that, being $B \subseteq S, u_{t}(y(s))>u_{t}(x(t))$ for almost every $s$ in $B$ and

$$
\begin{aligned}
\int_{B} \tilde{\gamma}(s)(x(s)-y(s)) d \mu(s) & =\sum_{i=1}^{k}\left[\int_{B_{i}}(x(s)-y(s)) d \mu(s)+\gamma_{i} \int_{S_{i} \cap T_{1}}(x(s)-y(s)) d \mu(s)\right] \\
& =\sum_{i=1}^{k}\left[\gamma_{i} \int_{S_{i} \cap T_{0}}(x(s)-y(s)) d \mu(s)+\gamma_{i} \int_{S_{i} \cap T_{1}}(x(s)-y(s)) d \mu(s)\right] \\
& =\int_{S} \gamma(s)(x(s)-y(s)) d \mu(s)=0 .
\end{aligned}
$$

This means that $(\tilde{\gamma}, B)$ is a fuzzy envied coalition for which, since $\left.\tilde{\gamma}\right|_{T_{0}} \in\{0,1\}$, negligible agents participate with their whole endowment.

Therefore, from the proposition above, being $\left.\gamma\right|_{T_{0}} \in\{0,1\}$ for any fuzzy envied coalition $(\gamma, S) \in \mathcal{F}$, in atomless economies the equivalence $F S F=S F$ is deduced by dispensing with the differentiability condition on the utility functions.

Corollary 4.2 In atomless exchange economies, an allocation is fuzzy strictly envy-free if and only if it is strictly envy-free. Thus, FSF $=S F$.

In the light of Corollary 4.2, the equivalence $W_{e i}=S F$ due to Zhou (1992) follows from our Theorem 3.4.

We now show that an allocation is competitive, and hence in the Aubin-core, if and only if almost every agent does not envy any fuzzy coalition with full support. In the literature a similar result is provided for the Aubin core (see Hervés-Beloso and Moreno-García 2001, 2008). In other words, let $\tilde{\mathcal{F}}$ denote the collection of fuzzy coalitions with full support and $F \tilde{S} F$ the corresponding set of fuzzy strictly fair allocations at which there is no envy towards coalitions in $\tilde{\mathcal{F}}$. Then, being $\tilde{\mathcal{F}} \subseteq \mathcal{F}$, one expects $F S F \subseteq F \tilde{S} F$. Actually, the next theorem shows that $F S F=F \tilde{\tilde{S}} F$ and hence, by Theorem 3.4 and Corollary 3.5, $W_{e i}=C_{A}=F S F=F \tilde{S} F$.

Theorem 4.3 Let $x$ be an allocation not fuzzy strictly envy-free, then any envious agent envies a fuzzy coalition with full support. Hence, $F \tilde{S} F=F S F$.

Proof Let $t$ be an arbitrary envious agent and let $(\gamma, S)$ be a fuzzy coalition envied by $t$ at $x$. Then, there exists an alternative allocation $y$ such that

(1) $u_{t}(y(s))>u_{t}(x(t))$ almost everywhere in $S$,

(2) $\int_{S} \gamma(s) y(s) d \mu(s)=\int_{S} \gamma(s) x(s) d \mu(s)$. 
We now show that condition (2) can be written with $\ll$, that is

$$
\left(2^{\prime}\right) \quad \int_{S} \gamma(s) y(s) d \mu(s) \ll \int_{S} \gamma(s) x(s) d \mu(s) .
$$

Indeed, first notice that since the commodity space is $\mathbb{R}_{++}^{\ell}, x$ as well as the alternative allocation $y$ is strictly positive. Now, for any $\varepsilon \in \mathbb{Q} \cap(0,1)$, where $\mathbb{Q}$ indicates the set of rational numbers, define the set $S_{\varepsilon}=\left\{s \in S: u_{t}(\varepsilon y(s))>u_{t}(x(t))\right\} \subseteq S$. Since $u_{t}$ is continuous, for any $s \in S$ there exists some $\varepsilon(s) \in \mathbb{Q} \cap(0,1)$ such that $s \in S_{\varepsilon(s)}$. Hence, $S$ is in the countable union of all the $S_{\varepsilon}$ with $\varepsilon \in \mathbb{Q} \cap(0,1)$. Since $\mu(S)>0$, there exists a certain $\varepsilon \in \mathbb{Q} \cap(0,1)$ such that $\mu\left(S_{\varepsilon}\right)>0$.

Define the allocation $z=\varepsilon y \chi_{S_{\varepsilon}}+y \chi_{S \backslash S_{\varepsilon}}+e \chi_{T \backslash S}$ and notice that, by (1), $u_{t}(z(s))>$ $u_{t}(x(t))$ for almost all $s \in S$. Furthermore, being $\gamma(s) y(s) \gg 0$ for almost all $s \in S_{\varepsilon}$, by (2) we have that

$$
\begin{aligned}
\int_{S} \gamma(s) z(s) d \mu(s)= & \int_{S \backslash S_{\varepsilon}} \gamma(s) y(s) d \mu(s)+\varepsilon \int_{S_{\varepsilon}} \gamma(s) y(s) d \mu(s) \\
& \ll \int_{S} \gamma(s) y(s) d \mu(s)=\int_{S} \gamma(s) x(s) d \mu(s) .
\end{aligned}
$$

Let $d \gg 0$ and $\alpha \in(0,1)$ be respectively such that $\int_{S} \gamma(s) z(s) d \mu(s)=$ $\int_{S} \gamma(s) x(s) d \mu(s)-d$ and $\alpha x(t) \mu(T \backslash S) \leqslant d$, and consider the allocation $z^{\prime}=$ $z \chi_{S}+(x+x(t)) \chi_{T \backslash S}$ and the fuzzy coalition $(\tilde{\gamma}, T)$ where $\tilde{\gamma}=\gamma \chi_{S}+\alpha \chi_{T \backslash S}$. Then, by monotonicity, $u_{t}\left(z^{\prime}(s)\right)>u_{t}(x(t))$ for almost all $s \in T$ and

$$
\begin{aligned}
\int_{T} \tilde{\gamma}(s) z^{\prime}(s) d \mu(s) & =\int_{S} \gamma(s) z(s) d \mu(s)+\int_{T \backslash S} \alpha(x(s)+x(t)) d \mu(s) \\
& =\int_{S} \gamma(s) x(s) d \mu(s)-d+\int_{T \backslash S} \alpha x(s) d \mu(s)+\alpha x(t) \mu(T \backslash S) \leqslant \int_{T} \tilde{\gamma}(s) x(s) d \mu(s) .
\end{aligned}
$$

To conclude the proof, let $K=\int_{T} \tilde{\gamma}(s)\left[x(s)-z^{\prime}(s)\right] d \mu(s) \geqslant 0$ and $\tilde{z}(s)=z^{\prime}(s)+$ $\frac{K}{\tilde{\gamma}(s)}$ for almost all $s \in T$. Then, by monotonicity, $u_{t}(\tilde{z}(s))>u_{t}(x(t))$ for almost all $s \in T$ and

$$
\int_{T} \tilde{\gamma}(s) \tilde{z}(s) d \mu(s)=\int_{T} \tilde{\gamma}(s) x(s) d \mu(s) .
$$

In the light of Theorem 4.3, as stated in the next corollary, an agent can belong simultaneously to the set of envious agents and to the envied coalition. This is because she is allowed to employ only a piece of her resources to join a coalition. Clearly, this possibility is excluded by the notion of strict fairness (Definition 2.3) for which an envious agent $t$ can not belong to the (crisp) envied coalition $S$, i.e. $t \notin S$.

Corollary 4.4 An agent can belong simultaneously to the set of envious agents and to the envied fuzzy coalition. 


\section{A further relation between fuzzy strict fairness and strict fairness}

In this section, we provide an interpretation of fuzzy coalitions and fuzzy strictly fair allocations based on associating to each mixed market an atomless economy. We employ the construction due to Greenberg and Shitovitz (1986) that assigns to a mixed market $\mathcal{E}=\left\{(T, \Sigma, \mu) ; \mathbb{R}_{++}^{\ell}, e,\left(u_{t}\right)_{t \in T}\right\}$ an atomless economy $\mathcal{E}^{*}=$ $\left\{\left(T^{*}, \Sigma^{*}, \mu^{*}\right) ; \mathbb{R}_{++}^{\ell}, e,\left(u_{t}\right)_{t \in T^{*}}\right\}$ obtained by splitting each atom $A$ of $\mathcal{E}$ into an atomless coalition $A^{*}$ with the same measure of $A$ and which is composed by a continuum of negligible agents of the same type of the atom $A .{ }^{12}$ The coalition $A^{*}$ is also called split atom $A$, and it is consistent with the interpretation of an atom as representative of a group of negligible agents (see Sect. 2). $T_{1}^{*}$ is the disjoint union of the split atoms $A^{*}$, whereas $\left(T^{*}, \Sigma^{*}, \mu^{*}\right)$ is the direct sum of $\left(T_{0}, \Sigma_{\left.\right|_{T_{0}}}, \mu_{\left.\right|_{T_{0}}}\right)$ and $T_{1}^{*}$ endowed with the Lebesgue measure. We can construct a one-to-one correspondence between $\mathcal{E}$ and $\mathcal{E}^{*}$ by means of two maps $\Phi$ and $\Psi$. Precisely, denoted by $X$ and $X^{*}$ respectively the set of allocations of the economies $\mathcal{E}$ and $\mathcal{E}^{*}$, the map $\Phi: X \rightarrow X^{*}$ assigns to each allocation $x \in X$ of $\mathcal{E}$ the unique allocation $\Phi(x) \in X^{*}$ of $\mathcal{E}^{*}$ given by $\Phi(x)=x \chi_{T_{0}}+\sum_{A \in T_{1}} x(A) \chi_{A^{*}}$. Conversely, the map $\Psi: X^{*} \rightarrow X$ assigns to each allocation $x^{*}$ of $\mathcal{E}^{*}$ the unique allocation $\Psi\left(x^{*}\right) \in X$ of $\mathcal{E}$ given by $\Psi\left(x^{*}\right)=x^{*} \chi_{T_{0}}+\sum_{A \in T_{1}} \frac{1}{\mu(A)} \int_{A^{*}} x^{*}(t) d \mu^{*}(t) \chi_{A}$. It can be proved that $\Psi(\Phi(x))=x$ for all $x \in X$. Moreover, if $x$ is efficient for $\mathcal{E}$, then $\Phi(x)$ is efficient for $\mathcal{E}^{*}$ and vice versa, if $x^{*}$ is efficient for $\mathcal{E}^{*}$, then $\Psi\left(x^{*}\right)$ is efficient for $\mathcal{E}$. The same one-to-one correspondence holds between the set of competitive allocations of the economies $\mathcal{E}$ and $\mathcal{E}^{*}$. A similar result is proved by Hüsseinov (1994), and further generalized by Hüsseinov and Pascoa (1997) to production economies, between the Aubin core of a finite economy and the core of the associated atomless economy. In what follows we prove that any fuzzy strictly fair allocation of $\mathcal{E}$ corresponds to a strictly fair allocation in $\mathcal{E}^{*}$ and vice versa. This allows us to interpret fuzzy coalitions and fuzzy strictly fair allocations of a mixed market respectively as standard coalitions and strictly fair allocations of the associated atomless economy $\mathcal{E}^{*}$. For this, we need that any strictly fair allocation in the atomless economy $\mathcal{E}^{*}$ satisfies the equal treatment property, meaning that agents with the same utility function receive, at a strictly fair allocation, indifferent bundles (see Lemma 3.5 in Donnini and Pesce (2020) for a stronger version of the equal treatment property of $S F$ in mixed markets). This property has its value as it states that identical agents are treated equally.

Lemma 5.1 Let $\mathcal{E}^{*}$ be an atomless economy, $x^{*}$ be a strictly fair allocation and $C$ be a coalition containing agents with the same utility function $u$. Then, $u\left(x^{*}(t)\right)=$ $u\left(\bar{x}^{*}(C)\right)$ for almost all $t \in C$, where $\bar{x}^{*}(C)=\frac{1}{\mu^{*}(C)} \int_{C} x^{*}(t) d \mu^{*}(t)$.

Proof Since $x^{*}$ is strictly fair the set $\left\{t \in C: u\left(\bar{x}^{*}(C)\right)>u\left(x^{*}(t)\right)\right\}$ has nullmeasure. Then, $u\left(\bar{x}^{*}(C)\right) \leqslant u\left(x^{*}(t)\right)$ for almost all $t \in C$. Define the set $D=\{t \in C$ : $\left.u\left(\bar{x}^{*}(C)\right)<u\left(x^{*}(t)\right)\right\}$, and assume to the contrary that $\mu^{*}(D)>0$. Let $\alpha=\frac{\mu^{*}(D)}{\mu^{*}(C)}$ and notice that $\alpha<1$, that is $\mu^{*}(C \backslash D)>0$, otherwise Lemma 2.4 would induce a contradiction. By the continuity of the utility function, there exist $\varepsilon \in(0,1)$ and

\footnotetext{
$\overline{12}$ Notice that the consumption set $\mathbb{R}_{++}^{\ell}$ and the total initial endowment $e$ are unchanged.
} 
a subset $E$ of $D$ with positive measure such that $u\left(\varepsilon x^{*}(t)\right)>u\left(\bar{x}^{*}(C)\right)$ for almost every $t$ in $E$. Hence, $u\left(\varepsilon \bar{x}^{*}(E)\right)>u\left(\bar{x}^{*}(C)\right)$ and $u\left(\bar{x}^{*}(C \backslash E)\right) \geqslant u\left(\bar{x}^{*}(C)\right)$.

Now, let $\beta=\frac{\mu^{*}(E)}{\mu^{*}(C)} \in(0,1)$ and notice that $\bar{x}^{*}(C)=\beta \bar{x}^{*}(E)+(1-\beta) \bar{x}^{*}(C \backslash E)$. Then,

$$
\begin{aligned}
u\left(\bar{x}^{*}(C)\right) & =u\left(\beta(1-\varepsilon) \bar{x}^{*}(E)+\beta \varepsilon \bar{x}^{*}(E)+(1-\beta) \bar{x}^{*}(C \backslash E)\right)> \\
& >u\left(\beta \varepsilon \bar{x}^{*}(E)+(1-\beta) \bar{x}^{*}(C \backslash E)\right) \geqslant u^{*}(\bar{x}(C)),
\end{aligned}
$$

which is a contradiction. Hence, $u\left(x^{*}(t)\right)=u\left(\bar{x}^{*}(C)\right)$ for almost all $t \in C$.

Theorem 5.2 Let $\mathcal{E}$ be a mixed market and $\mathcal{E}^{*}$ be the associated atomless economy.

(i) If $x$ is fuzzy strictly fair for $\mathcal{E}$, then $\Phi(x)$ is a strictly fair allocation for $\mathcal{E}^{*}$, i.e.

$$
x \in F S F \Rightarrow \Phi(x) \in S F
$$

(ii) If $x^{*}$ is a strictly fair allocation for $\mathcal{E}^{*}$, then $\Psi\left(x^{*}\right)$ is fuzzy strictly fair for $\mathcal{E}$, i.e.

$$
x^{*} \in S F \Rightarrow \Psi\left(x^{*}\right) \in F S F
$$

Proof $(i)$ Let $x$ be a fuzzy strictly fair allocation for $\mathcal{E}$. We already know that the corresponding allocation $\Phi(x)$ is efficient for $\mathcal{E}^{*}$. Assume to the contrary that $\Phi(x)$ is not strictly envy-free for $\mathcal{E}^{*}$. Then, by Lemma 2.4, given an arbitrary envious agent $t$ in $\mathcal{E}^{*}$, there exist a crisp coalition $S^{*}$ and an allocation $y^{*}$ such that $u_{t}\left(y^{*}(s)\right)>u_{t}(\Phi(x)(t))$ for almost every $s$ in $S^{*}$, and $\int_{S^{*}} y^{*}(s) d \mu^{*}(s)=$ $\int_{S^{*}} \Phi(x)(s) d \mu^{*}(s)$. Denote by $J=\left\{i: \mu^{*}\left(S^{*} \cap A_{i}^{*}\right)>0\right\}$, and consider for the economy $\mathcal{E}$, the coalition $S=\left(S^{*} \cap T_{0}\right) \cup \bigcup_{i \in J} A_{i}$ and the allocation $y=y^{*} \chi_{T_{0}}+\sum_{i \in J} \frac{1}{\mu^{*}\left(S^{*} \cap A_{i}^{*}\right)} \int_{S^{*} \cap A_{i}^{*}} y^{*}(t) d \mu^{*}(t) \chi_{A_{j}}+e \chi_{T \backslash S}$. By Lemma 2.4, $u_{t}(y(s))>u_{t}(x(t))$ for almost every $s$ in $S$. Define the fuzzy coalition $(\gamma, S)$ as $\gamma=1 \chi_{S^{*} \cap T_{0}}+\sum_{i \in J} \frac{\mu^{*}\left(S^{*} \cap A_{i}^{*}\right)}{\mu\left(A_{i}\right)} \chi_{A_{j}},{ }^{13}$ and note that

$$
\begin{aligned}
\int_{S} \gamma(s) y(s) d \mu(s) & =\int_{S \cap T_{0}} y(s) d \mu(s)+\sum_{i \in J} \gamma\left(A_{i}\right) y\left(A_{i}\right) \mu\left(A_{i}\right) \\
& =\int_{S^{*} \cap T_{0}} y^{*}(s) d \mu^{*}(s)+\sum_{i \in J} \frac{\mu^{*}\left(S^{*} \cap A_{i}^{*}\right) \mu\left(A_{i}\right)}{\mu\left(A_{i}\right) \mu^{*}\left(S^{*} \cap A_{i}^{*}\right)} \int_{S^{*} \cap A_{i}^{*}} y^{*}(s) d \mu^{*}(s) \\
& =\int_{S^{*}} y^{*}(s) d \mu^{*}(s)=\int_{S^{*}} \Phi(x)(s) d \mu^{*}(s) \\
& =\int_{S^{*} \cap T_{0}} \Phi(x)(s) d \mu^{*}(s)+\sum_{i \in J} \int_{S^{*} \cap A_{i}^{*}} \Phi(x)(s) d \mu^{*}(s) \\
& =\int_{S \cap T_{0}} \gamma(s) x(s) d \mu(s)+\sum_{i \in J} \gamma\left(A_{i}\right) x\left(A_{i}\right) \mu\left(A_{i}\right)=\int_{S} \gamma(s) x(s) d \mu(s) .
\end{aligned}
$$

\footnotetext{
13 Without loss of generality we may assume that $\gamma$ is simple because, as observed in Donnini and Pesce (2020), an envied coalition contains at most a finite number of atoms.
} 
Hence, $t$ envies $(\gamma, S)$ at $x$, meaning that any envious agent of $\mathcal{E}^{*}$ corresponds to an agent in $\mathcal{E}$ who envies a fuzzy coalition at $x$. Hence, $x$ is not fuzzy strictly envy-free, which is a contradiction.

(ii) Let $x^{*}$ be a strictly fair allocation for $\mathcal{E}^{*}$, then the associated allocation $\Psi\left(x^{*}\right)$ is efficient for $\mathcal{E}$. Assume to the contrary that $\Psi\left(x^{*}\right)$ is not fuzzy strictly envy-free for $\mathcal{E}$, meaning that for every envious agent $t$ in $\mathcal{E}$, there exist a fuzzy coalition $(\gamma, S)$ and an allocation $y$ such that

$$
\begin{aligned}
u_{t}(y(s)) & >u_{t}\left(\Psi\left(x^{*}\right)(t)\right) \text { for almost all } s \in S, \text { and } \\
\int_{S} \gamma(s) y(s) d \mu(s) & =\int_{S} \gamma(s) \Psi\left(x^{*}\right)(s) d \mu(s) .
\end{aligned}
$$

Note that if $t \in T_{0}, \Psi\left(x^{*}(t)\right)=x^{*}(t)$, whereas if $t=A \in T_{1}$, by Lemma 5.1, for almost all $t \in A^{*}$

$$
u_{t}\left(\Psi\left(x^{*}(t)\right)\right)=u_{t}\left(\frac{1}{\mu^{*}\left(A^{*}\right)} \int_{A^{*}} x^{*}(t) d \mu^{*}(t)\right)=u_{t}\left(x^{*}(t)\right)
$$

Hence,

$$
u_{t}\left(\Psi\left(x^{*}(t)\right)\right)=u_{t}\left(x^{*}(t)\right), \text { for almost all } t \in T^{*} .
$$

Define $J=\left\{j: A_{j} \subseteq S\right\}$, and consider the fuzzy coalition $\left(\gamma^{*}, S^{*}\right)$ in the atomless economy $\mathcal{E}^{*}$ given by $S^{*}=\left(S \cap T_{0}\right) \cup\left(A_{j}^{*}\right)_{j \in J}$, where each $A_{j}^{*}$ is the split atom $A_{j}$, and $\gamma^{*}=\gamma \chi_{S \cap T_{0}}+\sum_{j \in J} \gamma\left(A_{j}\right) \chi_{A_{j}^{*}}$.

Note that by construction $\mu(S)=\mu^{*}\left(S^{*}\right)$ and, given an envious agent $t$ in $\mathcal{E}$, by (5), (7) and by definition of $\Phi(y)$ we have that $u_{t}(\Phi(y)(s))>u_{t}\left(x^{*}(t)\right)$ for almost all $s \in S^{*}$. Furthermore, by (6)

$$
\begin{aligned}
\int_{S^{*}} \gamma^{*}(s) \Phi(y)(s) d \mu^{*}(s)= & \int_{S \cap T_{0}} \gamma^{*}(s) \Phi(y)(s) d \mu^{*}(s) \\
& +\sum_{j \in J} \int_{A_{j}^{*}} \gamma^{*}(s) \Phi(y)(s) d \mu^{*}(s) \\
= & \int_{S \cap T_{0}} \gamma(s) y(s) d \mu(s)+\sum_{j \in J} \int_{A_{j}} \gamma\left(A_{j}\right) y\left(A_{j}\right) d \mu(s) \\
= & \int_{S} \gamma(s) y(s) d \mu(s)=\int_{S} \gamma(s) \Psi\left(x^{*}\right)(s) d \mu(s) \\
= & \int_{S \cap T_{0}} \gamma(s) \Psi\left(x^{*}\right)(s) d \mu(s) \\
& +\sum_{j \in J} \int_{A_{j}} \gamma\left(A_{j}\right)\left(\frac{1}{\mu\left(A_{j}\right)} \int_{A_{j}^{*}} x^{*}(s) d \mu^{*}(s)\right) d \mu(s)
\end{aligned}
$$




$$
\begin{aligned}
& =\int_{S^{*} \cap T_{0}} \gamma^{*}(s) x^{*}(s) d \mu^{*}(s)+\sum_{j \in J} \int_{A_{j}^{*}} \gamma^{*}(s) x^{*}(s) d \mu^{*}(s) \\
& =\int_{S^{*}} \gamma^{*}(s) x^{*}(s) d \mu^{*}(s) .
\end{aligned}
$$

Then $x^{*}$ is not fuzzy strictly fair which, by Corollary 4.2, contradicts the fact that $x^{*}$ is strictly fair.

Corollary 5.3 Let $\mathcal{E}$ be a mixed market and $\mathcal{E}^{*}$ be the associated atomless economy. An allocation $x$ is fuzzy strictly fair in $\mathcal{E}$ if and only if $\Phi(x)$ is strictly fair in $\mathcal{E}^{*}$.

Proof In Theorem 5.2 the implication $x \in F S F \Rightarrow \Phi(x) \in S F$ has been already proved. For the converse, let $x$ be an allocation of the mixed economy $\mathcal{E}$ such that the corresponding allocation $\Phi(x)$ in the atomless economy $\mathcal{E}^{*}$ is strictly fair, i.e. $\Phi(x) \in S F$. By $(i i)$ of Theorem $5.2 \Psi(\Phi(x)) \in F S F$ and, being $x=\Psi(\Phi(x))$, $x \in F S F$.

Theorem 5.2(i) and Proposition 3.4 in Zhou (1992) allow us to provide an alternative proof of Theorem 3.4, obtaining the equivalence $W_{e i}=F S F$ as a corollary of Theorem 5.2 .

Corollary 5.4 If $u_{t}$ is differentiable for every $t$ in $T$, then $W_{e i}=F S F$.

Proof Let $x$ be a fuzzy strictly fair allocation of the mixed market $\mathcal{E}$. By Theorem $5.2(i), \Phi(x) \in S F$, which, in turn, by Proposition 3.4 in Zhou (1992), is an equalincome competitive allocation for the atomless economy $\mathcal{E}^{*}$. Finally, coming back to the economy $\mathcal{E}$, the allocation $\Psi(\Phi(x))$ is competitive and hence, being $\Psi(\Phi(x))=x$, we have that $x \in W_{e i}$, that is $F S F \subseteq W_{e i}$. Lemma 3.1 concludes the proof.

\section{Concluding remarks}

We have introduced a notion of fairness based on the fuzzy approach, which fully characterizes the set of equal-income competitive allocations in the general framework of mixed markets with no restriction on the measure space of agents. In what follows we conclude with some remarks and potential extensions of the fuzzy strict fairness notion.

Remark 6.1 In Donnini and Pesce (2020) we noted that in mixed markets an agent can envy only a finite number of non-negligible agents. This ensures that the fuzzy coalition (4) in the proof of Theorem $5.2(i)$ is well defined being simple. The requirement that fuzzy coalitions are identified by means of simple functions is not restrictive for the characterizations of competitive equilibria and the Aubin-core. Indeed, defining fuzzy coalitions as non-null $\mu$-integrable functions $\gamma: T \rightarrow[0,1]$, we get a subset $F S F$, because there are more coalitions to be potentially envied, and hence the equivalence in Theorem 3.4 a fortiori holds. 
Remark 6.2 Corollary 3.5 points out a fairness property of the Aubin-core, contrary to the (standard) core allocations which are neither fair in the sense of Foley (1967) (see Feldman and Kirman 1974). ${ }^{14}$ Actually, it is not clear if the core and the set FSF are comparable in mixed markets. In atomless economies they both coincide with the set of equal-income competitive allocations but, in general, they may differ. Indeed, on the one hand, in economies that fulfil the assumptions of Theorem $B$ of Shitovitz (1973), that is with at least two identical atoms, and in which agents' utility functions are not differentiable, it might be that $W_{e i}=C \varsubsetneqq F S F$. On the other hand, in economies with a single atom or several different atoms, and in which agents' utility functions are differentiable, it might be that $W_{e i}=F S F \varsubsetneqq C$.

Remark 6.3 Definition 2.1 can be extended to asymmetric information economies in which agents' private information is represented by a partition of the probability space describing the uncertainty, with the usual interpretation that agents are not able to distinguish states in the same event of their private information partition, and they are required to consume the same bundle in those indistinguishable states. A possible extension of fuzzy strict fairness in this context consists in imposing that an agent $t$ can envy a fuzzy coalition $(\gamma, S)$ only if members of $S$ receive the same private information signal of $t$ (see Basile et al. 2014; Donnini and Pesce 2020 for details). Proposition 3.1, Corollary 4.2 and Theorem 4.3 can be extended to asymmetric information economies, whereas the equivalence $W_{e i}=F S F$ of Theorem 3.4 fails even in atomless economies, because there might be fuzzy strictly fair allocations not supported by an equilibrium price (see Example 2.5 of Donnini and Pesce 2020).

Remark 6.4 A further generalization of $F S F$ consists in allowing agents to envy only their neighbours and get a local notion of fuzzy strict fairness. Formally, we can consider a covering of the set of agents $T$, that is a family $\mathcal{R}=\left\{C_{i}\right\}_{i \in I}$ of coalitions whose union equals $T$ and allow an agent $t$ of a certain class $C_{i}$ of $\mathcal{R}$ to envy a fuzzy coalition $(\gamma, S)$ only if $S$ is included in the same $C_{i}$ of $\mathcal{R}$. Each set $C_{i}$ of $\mathcal{R}$ can be indeed interpreted as the set of neighbours of $t$ or simply as the class of agents to whom $t$ directs her potential envy. In Donnini and Pesce (2021) we define two elements $C_{i}$ and $C_{j}$ of $\mathcal{R}$ to be connected if $\mu\left(C_{i} \cap C_{j}\right)>0$ and a covering $\mathcal{R}$ to be connected if for every pair of its elements there is a path linking up them, where a path is a sequence of elements of $\mathcal{R}$ connected to each other. With similar arguments used in Donnini and Pesce (2021), it can be proved that if $\mathcal{R}$ is connected, absence of envy among members of the same class $C_{i}$ of $\mathcal{R}$ is enough to ensure fairness in the whole economy $\mathcal{E}$, and thereby this local notion of fuzzy strict fairness coincides with $F S F$. It is worthwhile noting that, thanks to the fuzzy approach, this equivalence holds in mixed markets with no assumption on the space of agents as differently needed in Donnini and Pesce (2021).

Funding Open access funding provided by Università degli Studi di Napoli Federico II within the CRUICARE Agreement.

\footnotetext{
14 Feldman and Kirman (1974) present a three-agent economy in which the total initial endowment is equally divided among traders and the core allocation is not envy-free (see also Thomson 2011 and references cited therein).
} 
Open Access This article is licensed under a Creative Commons Attribution 4.0 International License, which permits use, sharing, adaptation, distribution and reproduction in any medium or format, as long as you give appropriate credit to the original author(s) and the source, provide a link to the Creative Commons licence, and indicate if changes were made. The images or other third party material in this article are included in the article's Creative Commons licence, unless indicated otherwise in a credit line to the material. If material is not included in the article's Creative Commons licence and your intended use is not permitted by statutory regulation or exceeds the permitted use, you will need to obtain permission directly from the copyright holder. To view a copy of this licence, visit http://creativecommons.org/licenses/by/4.0/.

\section{References}

Allouch N, Predtetchinski A (2008) On the non-emptiness of the fuzzy core. Int J Game Theory 37:203-210 Aubin J (ed) (1979) Mathematical methods of game economic theory. North-Holland, Amsterdam, New York, Oxford

Azrieli Y, Lehrer E (2007) On some families of cooperative fuzzy games. Int J Game Theory 36:1-15

Basile A, Graziano MG, Pesce M (2014) On fairness of equilibria in economies with differential information. Theory Decis 76:573-599

Basile A, Graziano MG, Tarantino C (2018) Coalitional fairness with participation rates. J Econ 123:97-139

Bhowmik A, Graziano MG (2015) On Vind's theorem for an economy with atoms and infinitely many commodities. J Math Econ 56:26-36

Cato S (2010) Local strict envy-freeness in large economies. Math Soc Sci 59:319-322

Donnini C, Pesce M (2020) Strict fairness of equilibria in asymmetric information economies and mixed markets. Econ Theory 69:107-124

Donnini C, Pesce M (2021) Absence of envy among "neighbors" can be enough. BE J Theor Econ 21(1):187204

Feldman A, Kirman A (1974) Fairness and envy. Am Econ Rev 64(6):995-1005

Florenzano M (1990) Edgeworth equilibria, fuzzy core and equilibria of a production economy without ordered preferences. J Math Anal Appl 153:18-36

Foley D (1967) Resource allocation and the public sector. Yale Econ Essays 7:45-98

Gabszewicz JJ, Mertens J (1971) An equivalence theorem for the core of an economy whose atoms are not "Too" Big. Econometrica 39:713-721

García-Cutrín J, Hervés-Beloso C (1993) A discrete approach to continuum economies. Econ Theory 3:577-583

Graziano MG, Pesce M, Urbinati N (2020) Generalized Coalitions and Bargaining Sets. J Math Econ 91:80-89

Greenberg J, Shitovitz B (1986) A simple proof of the equivalence theorem for oligopolistic mixed markets. J Math Econ 15:79-83

Hervés-Beloso C, Hervés-Estévez J, Moreno-García E (2018) Bargaining sets in finite economies. J Math Econ 74:93-98

Hervés-Beloso C, Moreno-García E (2001) The veto mechanism revisited. In: Lassonde M (ed) Approximation, optimization and mathematical economics. Phisica, Heidelberg, pp 147-157

Hervés-Beloso C, Moreno-García E (2008) Competitive equilibria and the grand coalition. J Math Econ 44:697-706

Hervés-Estévez J, Moreno-García E (2015) On restricted bargaining sets. Int J Game Theory 44:631-645

Hervés-Estévez J, Moreno-García E (2018a) A limit result on bargaining sets. Econ Theory 66:327-341

Hervés-Estévez J, Moreno-García E (2018b) Some equivalence results for a bargaining set in finite economies. Int J Econ Theory 14:129-138

Hildenbrand W (1974) Core and equilibria of a large economy. Princeton University Press, Princeton

Hüsseinov F (1994) Interpretation of Aubin's fuzzy coalitions and their extension. J Math Econ 23:499-516

Hüsseinov F, Pascoa MR (1997) Fuzzy cores in production economics. FEUNL Working Paper Series, no. 298, pp. 1-21

Liu J, Liu X (2014) Existence of Edgeworth and competitive equilibria and fuzzy cores in coalition production economies. Int J Game Theory 43:975-990

Liu J, Zhang H (2016) Coincidence of the Mas-Colell bargaining set and the set of competitive equilibria in a continuum coalition production economy. Int J Game Theory 45:1095-1109 
Mas-Colell A (1989) An equivalence theorem for a bargaining set. J Math Econ 18:129-139

Noguchi M (2000) A fuzzy core equivalence theorem. J Math Econ 34:143-158

Pesce M (2014) The veto mechanism in atomic differential information economies. J Math Econ 53:33-45

Shitovitz B (1973) Oligopoly in markets with a continuum of traders. Econometrica 41:467-501

Thomson W (1982) An informationally efficient equity criterion. J Public Econ 18:243-263

Thomson W (1988) A study of choice correspondences in economies with a variable number of agents. J Econ Theory 46:237-254

Thomson W (2011) Fair allocation rules. In: Arrow K, Sen A, Suzumura K (eds) Handbook of social choice and welfare. North-Holland, Amsterdam, New York

Thomson W, Zhou L (1993) Consistent solutions in atomless economies. Econometrica 61(3):575-587

Yang W, Liu J, Liu X (2011) Aubin cores and bargaining sets for convex cooperative fuzzy games. Int J Game Theory 40:467-479

Zhou L (1992) Strictly fair allocations in large exchange economies. J Econ Theory 57:158-175

Publisher's Note Springer Nature remains neutral with regard to jurisdictional claims in published maps and institutional affiliations. 